\title{
FURTHER TESTS OF THE VIOLATION OF $C P$ WITH NEUTRAL K MESONS
}

\author{
R. R. LEWIS \\ University of Michigan and Université de Genève \\ and \\ C. P. ENZ \\ Université de Geneve
}

Received 1 March 1965

The recent discovery [1] of the violation of $C P$ invariance in the decay $\mathrm{K}_{L} \rightarrow \pi^{+}+\pi^{-}$has stimulated a large number of proposals for the specific mechanism of $C P$ violation. In view of this and of the possibility that the $C P$ violation may be everywhere small, the empirical problem of verifying the many predictions appears very complex. In this letter we intend to consider the possibility of determining the additional parameters in the total mass matrix, irrespective of any specific assumptions about the mechanism. Three experiments are proposed, which if successful, would considerably clarify the problem of determining the origin of the $C P$ violations.

The analysis is based on the phenomenological description of neutral kaons [2-4], using a complex mass matrix $\Lambda=M-\frac{1}{2}$ i $\Gamma$. If $C P$ were valid, then, in a suitable representation, $\Lambda$ would be symmetric both along and across the diagonal, and would therefore have four real parameters. These parameters can be defined by the two complex eigenvalues $\lambda_{1}$ and $\lambda_{2}$, corresponding to the eigenvectors $\left|\mathbf{K}_{1}\right\rangle=\frac{1}{2} \sqrt{ } \mathbf{2}(|\mathbf{K}\rangle+|\overline{\mathbf{K}}\rangle)$ and $\left|\mathbf{K}_{2}\right\rangle=\frac{1}{2} \sqrt{ } 2(|\mathbf{K}\rangle-|\bar{K}\rangle$. In this representation $T$ implies symmetry across the diagonal and $T C P$ implies symmetry along the diagonal. The $C P$ violation can be described by the two additional complex parameters $r$ and $\rho$ where

$$
\begin{gathered}
r=\sqrt{\Lambda_{21} / \Lambda_{12}}, \quad \rho=\eta+\sqrt{1+\eta^{2}}, \\
\eta=\left(\Lambda_{22}-\Lambda_{11}\right) / 2 \sqrt{\Lambda_{12} \Lambda_{21}},
\end{gathered}
$$

defined so that $r \neq 1$ violates $T$ invariance and $\rho \neq 1$ violates $T C P$ invariance. The eigenvectors corresponding to the eigenvalues $\lambda_{\mathrm{S}, \mathrm{L}}=m_{\mathrm{S}, \mathrm{L}}+$ $-\frac{1}{2} i \gamma_{S, L}$ become

$\left|\mathrm{K}_{\mathrm{S}}\right\rangle=\frac{1}{2} \sqrt{2}(|\mathrm{~K}\rangle+\rho r|\overline{\mathbf{K}}\rangle), \quad\left|\mathrm{K}_{\mathrm{L}}\right\rangle=\frac{1}{2} \sqrt{2}(\rho|\mathbf{K}\rangle-r|\overline{\mathbf{K}}\rangle)$.

These states, together with their "inverse" states (not the ordinary Hermitian adjoints)

$$
\begin{aligned}
& \left\langle\widetilde{\mathrm{K}}_{\mathrm{S}}\right|=(\langle\mathbf{K}| r+\langle\overline{\mathbf{K}}| \rho) \sqrt{ } 2 / r\left(1+\rho^{2}\right), \\
& \left\langle\tilde{\mathbf{K}}_{\mathrm{L}}\right|=(\langle\mathbf{K}| \rho r-\langle\overline{\mathbf{K}}|)_{\sqrt{ }} / 2 / r\left(1+\rho^{2}\right)
\end{aligned}
$$

form a complete orthonormal set. The problem of measuring the widths $\gamma_{S}$ and $\gamma_{L}$ and the mass difference $\Delta m=m_{S}-m_{L}$ has been thoroughly discussed. The problem which we address is that of measuring $Z=1-r$ and $\zeta=1-\rho$; that is, of testing $T$ and $T C P$, and of measuring the magnitude and phase of the $C P$ admixture. (See ref. 3 for earlier discussion of this problem). Neither $Z$ nor $\zeta$ is known at present, since the only experimental evidence for $C P$ violation is a measurement of $\left|\left\langle 2 \pi\left|H_{\mathrm{W}}\right| \mathrm{K}_{\mathrm{L}}\right\rangle\right|^{2}$, which involves the decay amplitudes as well as $Z$ and $\zeta$.

We propose three experiments to determine $Z$ and $\zeta$. The first is simply a measurement of the total intensity of a neutral kaon beam versus the distance from the production vertex of a $K$ meson. As is well known, the intensity of $K$ and $\overline{\mathrm{K}}$ undergo damped oscillation. It was pointed out in ref. 2 that if $C P$ is violated the total intensity can also have a small oscillatory term, arising from the non-unitary character of the relation between $|\mathbf{K}\rangle,|\overline{\mathbf{K}}\rangle$ and $\left|\mathbf{K}_{\mathrm{S}}\right\rangle,\left|\mathbf{K}_{\mathbf{L}}\right\rangle$. We wish only to add the remark that the phase of this oscillatory term is fixed by $T$ or $T C P$ invariance. We find for the total intensity versus proper time

$A(\tau)=\frac{1}{2}\left\{\exp \left(-\gamma_{\mathrm{S}} \tau\right)[1-\operatorname{Re}(Z-\zeta)]+2 \exp -\frac{1}{2}\left(\gamma_{\mathrm{S}}+\gamma_{\mathrm{L}}\right) \tau\right\} \times$

$[\operatorname{Re} Z \cos \Delta m \tau+\operatorname{Im} \zeta \sin \Delta m \tau]+$

$$
\left.+\exp \left(-\gamma_{L} \tau\right)[1-\operatorname{Re}(Z+\zeta)]\right\}
$$

where only first order terms in $Z$ and $\zeta$ are retained. We see that $T$ implies an oscillation $\sim \sin (\Delta m \tau)$, whereas TCP implies an oscillation $\sim \cos (\Delta m \tau)$. We also note that the absence of an oscillatory term does not imply invariance 
under $C P, T$, or $T C P$, in first order.

Since $Z$ and $\zeta$ are expected to be of order $10^{-2}-10^{-3}$, the problem of observing such small oscillations appears to be very difficult. It is necessary to observe the strong interactions of $\mathbf{K}$ and $\overline{\mathbf{K}}$ along the beam, adding the two large amplitude oscillations to obtain the amplitude and phase of the resultant small oscillation. The difficulty of obtaining adequate statistical accuracy and of determining accurately the relative normalization $(\sigma \mathrm{K} / \sigma \overline{\mathrm{K}})$ appear to us insurmountable.

It is therefore important to realize that the same information can be obtained in a different way: by measuring instead the $\mathrm{K}$ intensity in a mixed beam in which exactly equal numbers of $\mathrm{K}$ and $\overline{\mathbf{K}}$ are initially produced. We find

$P(\tau)=\frac{1}{4}\left\{\exp \left(-\gamma_{\mathrm{S}} \tau\right)[1+\operatorname{Re}(Z+\zeta)]+\right.$

$-2 \exp \left[-\frac{1}{2}\left(\gamma_{S}+\gamma_{L}\right) \tau\right][\operatorname{Re} Z \cos \Delta m \tau-\operatorname{Im} \zeta \sin \Delta m \tau]+$

$$
\left.+\exp \left(-\gamma_{\mathcal{L}} \tau\right)[1+\operatorname{Re}(Z-\zeta)]\right\} \text {. }
$$

One possibility for measuring this is the double charge exchange scattering of charged $\mathrm{K}$ mesons, $\mathrm{K}^{+} \rightarrow$ neutral $\mathrm{K} \rightarrow \mathrm{K}^{+}$and $\mathrm{K}^{-} \rightarrow$ neutral $\mathrm{K} \rightarrow \mathrm{K}^{+}$, as function of the proper time delay. Equal numbers of initial $\mathrm{K}^{+}$and $\mathrm{K}^{-}$events must be obtained and the results added. Another possibility is detection of the strong interaction of kaons produced in $\mathrm{K} \overline{\mathrm{K}}$ pairs, adding the observed numbers of strong interactions of $\mathrm{K}$ mesons irrespective of how the associated meson interacts or decays [5]. In this case the $K$ cross section need not be accurately known and the main problem is statistical. Such relationships between $P(\tau)$ and $A(\tau)$ are analogous to the well-known relationship between polarization and asymmetry of spin $\frac{1}{2}$ beams, since calculations with the mass matrix $\Lambda$ are formally equivalent to the polarization theory of spin $\frac{1}{2}$ particles.

The next experiment is one which is insensitive to $T$ and $T C P$ invariance but will determine the magnitude of $\rho r$. It consists of measuring the relative intensity of any partial decay rate in a "stale" beam of neutral kaons in which a $K$ or a $\bar{K}$ is initially produced. Thus if $3 \pi$ decays are measured after proper time $\tau$, the ratio is

$$
\begin{aligned}
& R(\tau)=\left|\begin{array}{l}
\left\langle 3 \pi\left|H_{\mathrm{W}}\right| \mathbf{K}_{\mathrm{L}}\right\rangle \exp \left(-\mathrm{i} \lambda_{\mathrm{L}} \tau\right)\left\langle\tilde{\mathbf{K}}_{\mathrm{L}} \mid \mathbf{K}\right\rangle \\
\left.\hdashline 3 \pi\left|H_{\mathrm{W}}\right| \mathbf{K}_{\mathrm{L}}\right\rangle \exp \left(-\mathrm{i} \lambda_{\mathrm{L}} \tau\right)\left\langle\tilde{\mathbf{K}}_{\mathrm{L}} \mid \overline{\mathbf{K}}\right\rangle
\end{array}\right|^{2}= \\
& =\left|\frac{\left\langle\tilde{\mathbf{K}}_{L} \mid \overline{\mathbf{K}}\right\rangle}{\left\langle\widetilde{\mathrm{K}}_{\mathrm{L}} \mid \overline{\mathbf{K}}\right\rangle}\right|^{2}=|\rho r|^{2} \text {. }
\end{aligned}
$$

A value of $R$ different from unity is another viola- tion of $C P$ and gives useful information on the values of $Z$ and $\zeta$. This is a special case of the effect of $C P$ violations on the decay curve of eacl channel. At earlier $\tau$, both $\mathrm{K}_{\mathrm{S}}$ and $\mathrm{K}_{\mathrm{L}}$ contribute and the results depend on the decay amplitudes for that channel. This result has the advantage of being independent of the decay amplitudes and the detection efficiency.

The last experiment is one sensitive to the phase of $Z$ and $\zeta$. It consists of attempting to cancel the intrinsic $C P$ admixture in $\mathrm{K}_{\mathrm{S}}, \mathrm{K}_{\mathrm{L}}$, by the induced $C P$ admixture resulting from coherent scattering in a regenerator. The scatterir phases are assumed known from scattering and coherent regeneration measurements, and they provide a phase standard from which the phases of $Z$ and $\zeta$ can be determined.

The effect of a scattering medium on the coherent kaon beam [6] is accounted for by addition to $\Lambda$ of an "effective mass" matrix $\Lambda_{\text {coh }}=$ $=-\frac{2 \pi \mathrm{N}}{M}\left|\begin{array}{ll}f & \mathrm{o} \\ \circ & \bar{f}\end{array}\right|$, where $N$ is the number density of scattering centers and $f, \bar{f}$ are the forward scattering amplitudes of $\mathrm{K}, \overline{\mathrm{K}}$. The eigenvectors of $\Lambda^{\prime}=\Lambda+\Lambda_{\text {coh }}$ can be expressed in terms of $Z$, $\zeta$ and a single additional parameter $\delta=$ $=\pi N(f-\bar{f}) / M\left(\lambda_{\mathrm{S}}{ }^{-\lambda_{\mathrm{L}}}\right)$. If only terms of first order in $\delta, Z, \zeta$ are retained, we find

and

$$
\left|\mathbf{K}_{\mathbf{S}}^{\prime}\right\rangle \approx \frac{1}{2}, / 2[(1-\delta)|\mathbf{K}\rangle+(1-Z-\zeta+\delta)|\bar{K}\rangle]
$$

$$
\left|\mathbf{K}_{\mathbf{L}}^{\prime}\right\rangle \approx \frac{1}{2}, / 2[(1+\delta-\zeta)|\mathbf{K}\rangle-(1-Z-\delta)|\overline{\mathbf{K}}\rangle]
$$

which gives for the $C P$ admixtures

$$
\left\langle\mathbf{K}_{2} \mid \mathbf{K}_{\mathbf{S}}^{\prime}\right\rangle \approx \frac{1}{2}(Z+\zeta)-\delta, \quad\left\langle\mathbf{K}_{1} \mid \mathbf{K}_{\mathbf{L}}^{\prime}\right\rangle \approx \frac{1}{2}(Z-\zeta)+\delta .
$$

Any experiment measuring the $C P$ violation of the coherent beam of kaons in the medium will involve these parameters rather than $Z$ and $\zeta$ alone and will enable determination of the phase of $Z$ and $\zeta$ relative to $\delta$. We note that the results will also test $T$ and $T C P$, since if $T C P$ is valid $(\zeta=0)$ the vanishing of $\left\langle K_{1} \mid K_{L}^{\prime}\right\rangle$ is accompanied by the doubling of $\left\langle\mathbf{K}_{2} \mathbf{K}_{\mathbf{S}}^{\prime}\right\rangle$, whereas if $T$ is valid $(Z=0)$ both $\left\langle\mathbf{K}_{\mathbf{1}} \mid \mathbf{K}_{\mathrm{L}}^{\prime}\right\rangle$ and $\left\langle\mathbf{K}_{\mathbf{2}} \mid \mathbf{K}_{\mathbf{S}}\right\rangle$ vanish togethe Similar conclusions are possible even if the $C P$ admixture is not exactly cancelled.

To discuss an experiment more specifically, we will make the additional assumption that $C P$ is not violated in the $\pi^{+}+\pi^{-}$decay amplitudes. Inclusion of a small $C P$ violation in the decay am plitudes will weaken the conclusions but will not destroy the effect. Then the measured rate of $\mathrm{K}_{\mathrm{L}} \rightarrow \pi^{+}+\pi^{-}$determines the magnitude of the $C P$ admixture in $\mathrm{K}_{\mathrm{L}}$,

$$
\left|\left\langle\mathbf{K}_{1} \mid \mathbf{K}_{\mathrm{L}}\right\rangle\right|^{2} \approx \frac{1}{4}|Z-\zeta|^{2} \approx 2 \times 10^{-3}
$$


Similarly, coherent regeneration experiments can determine the phase and magnitude of $\delta$. It is important to note that for common metals $(\mathrm{Al}, \mathrm{Cu}$, Fe) $|\delta| \approx 2 \times 10^{-2}$. This implies that the critical density at which $|\delta|=\frac{1}{2}|Z-\zeta|$ is at $\sim \frac{1}{10}$ of the normal density, and that therefore a spark chamber (or a heavy liquid bubble chamber) with variable density provides an ideal medium for such experiments. It is suggested that the $2 \pi$ and $3 \pi$ decay modes of $K_{S}^{\prime}$ and $K_{L}^{\prime}$ be measured versus density in such a medium. It is also important to include this effect in the analysis of $\mathrm{K}_{13}$ decays in propane, in testing the $\Delta S / \Delta Q$ rule.

A more detailed article on these topics is in preparation. The first author (RRL) whishes to sincerely thank Prof. J. M. Jauch for the hos- pitality extended at the Institut de Physique Theorique, where this work was performed.

\section{References}

1. J.H.Christenson, J.W.Cronin, V.L.Fitch and R. Turlay, Phys. Rev. Letters 13 (1964) 138; A. Abashian, R. J.Abrams, D.W. Carpenter, G.P. Fisher, B.M.K. Nefkens and J.H.Smith, Phys.Rev. Letters 13 (1964) 243.

2. T.D. Lee, Reinhard Oehme and C.N.Yang, Phys. Rev. 106 (1957) 340.

3. R.G.Sachs, Phys.Rev. 129 (1964) 2280; Annals Phys. 22 (1963) 239.

4. Tai Tsun Wu and C.N.Yang, Phys. Rev. Letters 13 (1964) 380 .

5. A more thorough treatment of the effect of $C P$ violation in the $K \mathrm{~K}$ system will be published later.

6. Myron L.Good, Phys. Rev. 106 (1957) 591.

\section{A POSSIBLE $Y=2, S=0$ pp $\pi^{+}$RESONANCE AT $2520 \mathrm{MeV}$}

\section{J. KIDD, L. MANDELLI, V. PELOSI, S. RATTI, A. SICHIROLLO and L. TALLONE *}

Istituto di Fisica e Sezione I.N.F.N., Milano

and

F. CONTE and G. TOMASINI

Istituto di Fisica e Sezione I.N.F.N., Genova

Received 17 March 1965

In this letter we present evidence which can be interpreted as indication of a $\mathrm{pp} \pi^{+}$resonance at $2520 \mathrm{MeV}$, with a full width of about $120 \mathrm{MeV}$.

A systematic study of $\mathrm{pp}$ interactions at $4 \mathrm{GeV} / c$ has been carried out in the $81 \mathrm{~cm}$ Saclay Hydrogen Bubble Chamber exposed to the CERN P.S. Preliminary results on partial channels have been already given [1].

In the present letter we are dealing with 929 events fitted by the 4 constraint reaction:

$$
p+p \rightarrow p+p+\pi^{+}+\pi^{-}
$$

among 2070 4-prong events measured to date. Remeasurement and independent kinematical analysis [2] of a partial sample of events and measurements of ionization by gap counting on tracks with ambiguous mass assignments ensure us against fitting-procedure losses and contamination

\footnotetext{
* Now at CERN, Geneva.
}

from other channels (overall level of confidence greater than $97 \%$ ).

The distribution of the square of the missing mass peaked at $M^{2}=-0.5 \times 10^{-3}(\mathrm{GeV})^{2}$ with a half width at half maximum of $\frac{1}{2} \Gamma=1.3 \times 10^{-3}$ $\mathrm{GeV})^{2}$ gives an idea of our measuring accuracy. We feel that we are dealing with a rather unbiased sample of events representing reaction (1), corresponding to a cross section:

$$
\sigma=(2.95 \pm 0.15) \mathrm{mb} .
$$

The reaction is strongly dominated by the production of the $\left(\frac{3}{2}, \frac{3}{2}\right)$ isobar. About $60 \%$ of isobar production gives the best fit to the distribution of the $M^{*}{ }_{\mathrm{p}}{ }^{+}$combinations.

In fig. 1 are plotted the distributions of the following effective masses:
a) $M_{\pi \pi}^{*}$
c) $M_{\mathrm{pp} \pi^{+}}^{*}$
b) $M_{\mathrm{p} \pi \pi}^{*}$ (two combinations per event):
d) $M_{\mathrm{pp} \pi}^{*}$ 